\title{
AOR
}

Selected Papers of \#AolR2021:

The 22nd Annual Conference of the

Association of Internet Researchers

Virtual Event / 13-16 Oct 2021

\section{TAKING THE REDPILL: TALKING ABOUT EXTREMISM}

Alice E. Marwick

Department of Communication; Center for Information, Technology, \& Public Life, University of North Carolina at Chapel Hill

Katie Furl

Department of Sociology; Center for Information, Technology, \& Public Life, University of North Carolina at Chapel Hill

\section{Introduction}

There is great public concern about "online radicalization," the process by which individuals are exposed to extremist internet content, adopt extremist or hateful ideas, and commit acts of political violence. This concept was popularized in the post-9/11 context of state concerns about Islamic extremism and used to justify military intervention and surveillance of Muslim communities around the globe (Kundnani, 2012). More recently, "online radicalization" describes the adoption of far-right and fringe beliefs such as white nationalism, male supremacy, or conspiracy theories, following internet exposure to them (Roose, 2019).

Radicalization is an inherently problematic concept. It frequently conflates adopting extremist viewpoints with committing political violence. It seeks to intervene on a universal path to extremism, yet a large body of research has found no common characteristics or paths to radicalization. And as a concept formulated in a context of Orientalism, which assumes that to study the radical is to study the other it fails to take into account the fundamental American roots of white supremacy and racism (Neiwert, 2017; Stern, 2019).

Most relevant to AOIR, while the role of the internet in spreading fringe and far-right beliefs is undeniable, often the political, economic, and emotional complexities that draw people to them are ignored in favor of simplistic narratives that suggest that exposure to YouTube, Parler, or 4chan causes people to adopt extremist mindsets. This is a version of the (somewhat mythic) "magic bullet" theory of media effects which presumed that exposure to a media message led to adoption of it (Sproule, 1989). There is little integration of internet or media studies into studies of radicalization. 
To understand the adoption of fringe and far-right beliefs outside the problematic frame of "radicalization", this paper takes up narratives of "redpilling," slang for coming to believe something counterfactual to mainstream consensus. The "red pill" refers to the scene in The Matrix in which the character Morpheus offers the protagonist Neo the option of taking a red or blue pill. By taking the red pill, Neo sees "the truth" about the Matrix.

This project draws from qualitative and ethnographic research on online far-right subcultures (Daniels, 2009; Lewis, 2018; Munn, 2019; Phillips, 2015) and critical terrorism studies (Conway, 2017; Stampnitzky, 2013). We asked three descriptive questions to find out how members of far-right and fringe communities understand and perform their own community enrollment through the term "redpilling":

RQ1: How is the term "redpilling" used by members of fringe and far-right communities?

RQ2: What things do people claim to be redpilled by?

RQ3: How do members describe their own redpilling experiences?

\section{Method}

We conducted critical discourse analysis and qualitative data analysis using a grounded theory method to answer these research questions.

First, we assembled a broad corpus of text discussions of "redpilling" in situ, primarily in internet spaces devoted to far-right and fringe communities, using publicly available English-language datasets collected by other researchers. We used pushshift.io to search 14 subreddits ( $r / T h e R e d P i l l, r /$ WhiteNationalism, etc.) and Gab.ai (Fair \& Wesslen, 2019); leaked Discord chats of white nationalist groups obtained by antifascist activists Unicorn Riot; and publicly available datasets of Parler (Aliapoulios et al., 2021), Telegram (Baumgartner et al., 2020), and 4chan's /pol board (Papasavva et al., 2020). ${ }^{1}$

We searched for discussions where the term "redpill," "redpilled" "rabbit hole" or "rabbithole" appeared. We then cleaned the search results to remove irrelevant instances and imported the resulting data into MaxQDA. We used a subset of the Reddit and Gab data to generate a codebook and taxonomy of "redpilling content." We conducted qualitative coding to closely examine the data, develop categories, and identify matters for future analytic consideration (Saldana, 2009).

\section{Findings}

We find the following in relation to our 3 research questions:

RQ1: How is the term "redpilling" used?

\footnotetext{
${ }^{1}$ There are ethical considerations in using some of these datasets, particularly those which were obtained by surreptitious means. As a result, we removed all usernames and identifying information from the dataset.
} 
Redpilling is used to describe the process of being converted to a new way of thinking; as an adjective exemplifying alt-right (or Qanon, or anti-feminist) views; and as a noun describing a fact that "proves" redpilled thinking. In conspiratorial communities, "going down the rabbithole" is more commonly used, demonstrating the centrality of "doing one's own research" to community participation.

$R Q 2:$ What are people redpilled by?

People mentioned specific media texts (Death of the West by Pat Buchanan, the video series Fall of the Cabal); influencers (Alex Jones, Lauren Southern); ideologies (feminism); events (COVID, the 2020 Black Lives Matter protests); and being exposed to knowledge/facts that changed their mind about a social or political matter. They were also redpilled by family members, friends, and personal events. Anti-feminist redpilling is prompted more by interpersonal interactions than racist or anti-Semitic redpilling, which often relies more on media or influencer interactions.

RQ3: How do people describe redpilling?

Although redpilling is imagined as a dramatic shift, it was usually discussed more as an ongoing process in which people gradually came to take on a specific ideology. While redpilling was frequently construed as both a necessary and difficult truth, those who consider themselves redpilled often used redpilling to justify prejudice and appreciated how redpill narratives provided simplistic, explanatory frameworks for understanding complex social issues.

\section{Discussion}

Analysis suggests that "online radicalization" is an ongoing process in which people come to believe extremist viewpoints by consuming far-right content, participating in farright internet spaces, viewing interpersonal interactions through an ideological lens, and interacting with friends and family with similar views. Thus, it resembles typical processes of socialization into an internet community or social movement. We hope that our findings contribute to more nuanced understandings of the spread of far-right and fringe ideas, with the goal of decreasing their dissemination and influence.

\section{References}

Aliapoulios, M., Bevensee, E., Blackburn, J., De Cristofaro, E., Stringhini, G., \& Zannettou, S. (2021). An Early Look at the Parler Online Social Network. ArXiv Preprint ArXiv:2101.03820.

Baumgartner, J., Zannettou, S., Squire, M., \& Blackburn, J. (2020). The Pushshift Telegram Dataset. Proceedings of the International AAAI Conference on Web and Social Media, 14, 840-847.

Conway, M. (2017). Determining the role of the internet in violent extremism and terrorism: Six suggestions for progressing research. Studies in Conflict \& Terrorism, 40(1), 77-98. 
Daniels, J. (2009). Cyber racism: White supremacy online and the new attack on civil rights. Rowman \& Littlefield.

Fair, G., \& Wesslen, R. (2019). Shouting into the void: A database of the alternative social media platform gab. Proceedings of the International AAAI Conference on Web and Social Media, 13, 608-610.

Kundnani, A. (2012). Radicalisation: The journey of a concept. Race \& Class, 54(2), 325. https://doi.org/10.1177/0306396812454984

Lewis, R. (2018). Alternative Influence: Broadcasting the Reactionary Right on YouTube. Data \& Society Research Institute. https://datasociety.net/wpcontent/uploads/2018/09/DS_Alternative_Influence.pdf

Munn, L. (2019). Alt-right pipeline: Individual journeys to extremism online. First Monday, 24(6).

Neiwert, D. (2017). Alt-America: The rise of the radical right in the age of Trump. Verso Books.

Papasavva, A., Zannettou, S., De Cristofaro, E., Stringhini, G., \& Blackburn, J. (2020). Raiders of the lost kek: 3.5 years of augmented 4chan posts from the politically incorrect board. Proceedings of the International AAAI Conference on Web and Social Media, 14, 885-894.

Phillips, W. (2015). This Is Why We Can't Have Nice Things: Mapping the Relationship Between Online Trolling and Mainstream Culture. MIT Press.

Roose, K. (2019, June 8). The Making of a YouTube Radical. The New York Times. https://www.nytimes.com/interactive/2019/06/08/technology/youtube-radical.html

Saldana, J. (2009). The coding manual for qualitative researchers. Sage Publications, Inc.

Sproule, J. M. (1989). Progressive propaganda critics and the magic bullet myth. Critical Studies in Media Communication, 6(3), 225-246.

Stern, A. M. (2019). Proud Boys and the White Ethnostate: How the Alt-Right is Warping the American Imagination. Boston: Beacon Press.

Stampnitzky, L. (2013). Disciplining Terror: How Experts Invented "Terrorism." Cambridge University Press. 
